\title{
Automatic Target Classification in Passive ISAR Range-Crossrange Images
}

\author{
Andrea Manno-Kovacs*, Elisa Giusti ${ }^{\dagger}$, Fabrizio Berizzi ${ }^{\dagger \ddagger}$ and Levente Kovács* \\ *Machine Perception Research Lab, Institute for Computer Science and Control - MTA SZTAKI \\ Hungarian Academy of Sciences, Budapest, Hungary \\ Email: \{andrea.manno-kovacs, levente.kovacs\}@sztaki.mta.hu \\ ${ }^{\dagger}$ Department of Information Engineering, University of Pisa, Italy \\ Email: elisa.giusti@cnit.it

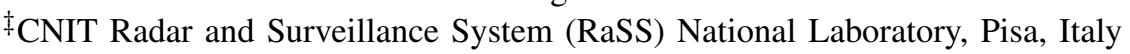 \\ Email: f.berizzi@iet.unipi.it
}

\begin{abstract}
This paper presents a method for automatic analysis of passive radar 2D ISAR images to evaluate the possibilities and capabilities of image feature based target extraction and classification. The goal is to extend signal processing based detection and recognition methods with image information. The presented method is fast, easily embeddable and extendable, works near real-time, and we show its viability for classification using real passive 2D ISAR images.
\end{abstract}

\section{INTRODUCTION}

Passive radar systems use one or more non-cooperative illuminators of opportunity (e.g., digital video broadcasts [1], mobile communications [2], digital or FM radio [3], etc.) as signal sources and one or more controlled receivers. Passive radars have recently received a renewed interest from the scientific community since the recent technological advances have made the realization of low cost passive radars [4] and real time processing possible. Following the recent technological advances on this field, additional radar techniques are added to passive radars to make them able to handle several tasks. One of such task is the radar imaging [5] of non-cooperative targets though the use of Inverse Synthetic Aperture Radar (ISAR) methods [6], [7], [8], which in turn may open the door to Automatic Target Classification (ATC). Although recent researches have demonstrated the feasibility of passive radar imaging, the ability to use these ISAR images for target recognition was formulated but not demonstrated. This paper is an attempt to prove whether 2D ISAR passive radar images can be use for such a purpose.

In particular, this paper focuses on target segmentation and classification using 2D ISAR range-crossrange images of passive radar systems [9]. The goal of the proposed method is to have a generic, model-free approach for image-based target recognition that can be used for various target classes and image resolutions, can be used with a low number of target samples, but can be easily extended to support larger target classes. The most important application area is silent, passive defense observation for force and area protection (e.g., [4]).

Passive radar technology has been applied to target detection and imaging [2], [7] and for target classification [10], [11], [12] using signal or image processing approaches. Our goal is to extract targets and features from $2 \mathrm{D}$ passive ISAR images originating from a multistatic passive radar measurement system that can be used for image-based classification. When we know possible target structures, works like [12], [13] provide detection methods using a Markovian approach.

However, our goal is to detect targets without target model constraints. The contribution of the current work is that it proposes a lightweight solution, without the need of periodic retraining, that can also work with a low number of examples. The proposed method produces a segmentation of the target from 2D passive ISAR images, based on previous results in saliency based feature map generation [14], [15]. First, we produce a fused feature map of directional and textural salient information, then we extract target regions and their contours as a basis for classification using shape based recognition and retrieval [16].

The proposed approach has been tested on data acquired with the SMARP (Software-defined Multiband Array Passive Radar) passive radar demonstrator. SMARP has been developed by the Radar and Surveillance Systems Laboratory (RaSS Lab.) of the Italian National Inter-University Consortium for Telecommunications (CNIT). SMARP is a dual band and dual polarization passive radar operating at UHF $(470790 \mathrm{M} \mathrm{Hz})$ and S-band $(2,1002,200 M H z)$. In its current version SMARP is able to acquire up to $25 \mathrm{MHz}$ bandwidth signal at UHF [4].

A picture of SMARP and some detection and tracking results are shown in Fig. 11(a)-(b). Examples of Passive ISAR images obtained by using the SMARP system are shown in Fig. 2. Conversely from conventional ISAR images which are in the range/Doppler domain, such images are in a fully spatial coordinate system. To get the ISAR images in the range/crossrange domain, the algorithm proposed in [17] has been applied in order to scale cross-range axis from $\mathrm{Hz}$ to $\mathrm{m}$.

\section{THE PROPOSED APPROACH}

The main goal of the approach proposed in this paper is to provide a method that can work with a limited dataset, but can scale to hundreds or thousands of samples as well. The method has two steps: i). detection and extraction of targets from range-crossrange images, along with target features, and ii). classification of the extracted target based on previously seen samples. 


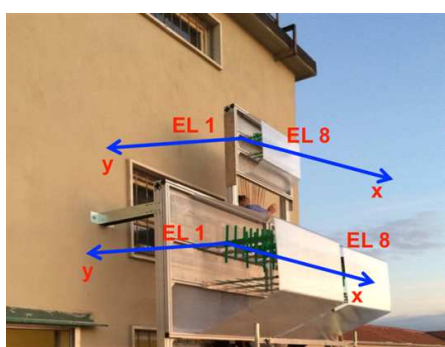

(a)

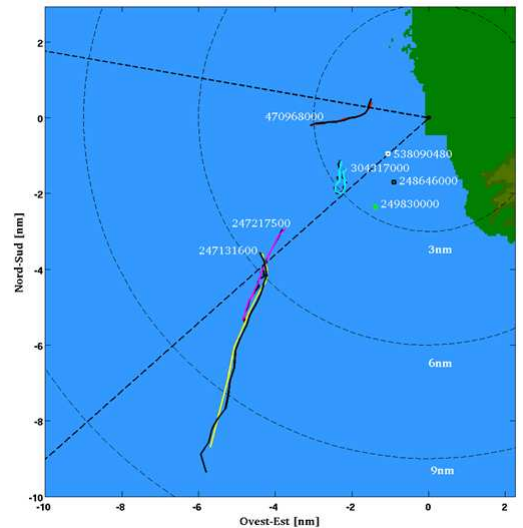

(b)

Fig. 1. (a) Picture of the SMARP system and (b) detection and tracking results. Colored lines are the targets AIS trajectories of ships and the black lines are the radar tracks.

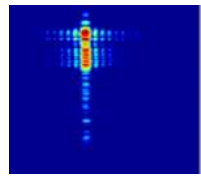

(a)

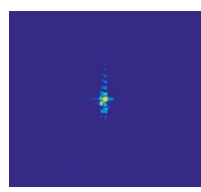

(e)

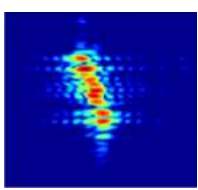

(b)

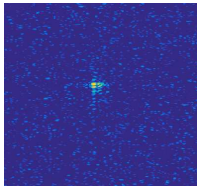

(f)

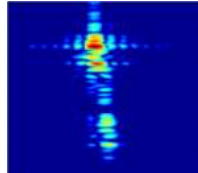

(c)

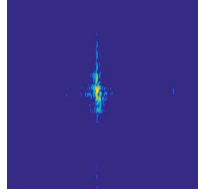

(g)

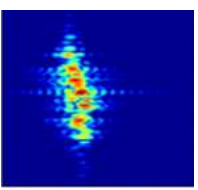

(d)

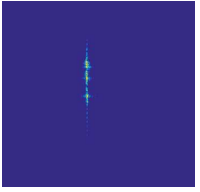

(h)
Fig. 2. Example raw input range-crossrange images.

The dataset that we used for processing and testing contains real range-crossrange images produced by passive ISAR measurements, and were provided by partners of the MAPIS (Multichannel passive ISAR imaging for military applications) Project Consortium. The dataset contains images of targets from 8 classes, 128 images in total. Figs. 2 and 3 show examples of input images that we process for detection and classification.

\section{A. Detection and Extraction}

The goal of the first step is the detection of objects/targets in obtained range-crossrange images. The method that we propose is generic, in the sense that does not use any a priori target information (shape, model, etc.), but relies only on discriminative image features. A benefit of such an ap-

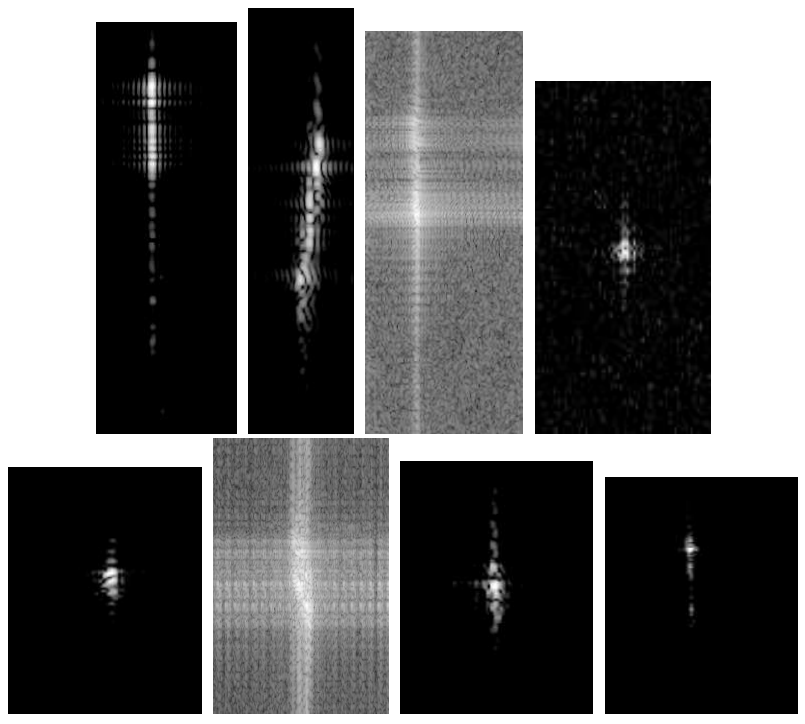

Fig. 3. Example input range-crossrange images resized according to real size ratios (having different resolutions).

TABLE I. RESOLUTIONS (METERS/PIXEL) OF THE VARIOUS RAW INPUT IMAGES, AND THEIR AVERAGE WIDTH/HEIGHT (CROSSRANGE-RANGE) VALUES (METERS).

\begin{tabular}{|c|c|c|c|}
\hline Class & Im. resolution (m/px) & Avg. im. width (m) & Avg. im. height (m) \\
\hline \hline A & $1.44-3.12$ & 1767 & 953 \\
\hline B & $0.82-1.58$ & 118 & 452 \\
\hline C & $0.91-2.35$ & 432 & 706 \\
\hline D & 0.81 & 212 & 470 \\
\hline E & $3.1-3.14$ & 1126 & 1018 \\
\hline F & $4.65-4.69$ & 661 & 1524 \\
\hline G & $1.3-1.32$ & 636 & 427 \\
\hline H & $4.18-4.21$ & 5946 & 1371 \\
\hline
\end{tabular}

proach is flexibility and independence from possible target model constraints. The goal here is the detection of the target candidates, and the extraction of features that can later be used for classification and recognition. The method that we propose is based on the extraction of fused morphological, textural and edge feature maps. The final features that we aim to extract and retain are the shape/contour of the target and its length.

Input images can be of various size and resolution, and they can contain targets with different meters/pixel resolution (e.g., Table. II shows the various resolutions from the used dataset). As a first step, we resize the raw inputs to have a ratio consistent with their resolution (e.g., Fig. 3). In Fig. 4 the raw input image is shown in the first row, the resized image is in Fig. 4(a), representing a 103.28 meter $\times 512.39$ meter area. The resized image is processed using the proposed algorithm.

The main concept of the proposed detection and extraction is to first extract the salient object - target candidate - in the image, then use the features of this object in the classification step. As a first step of the object extraction, a texture map is calculated by using the sparse texture model of [18] and measuring the statistical textural distinctiveness of the occurring texture atoms. After extracting rotation-invariant, neighborhood-based textural representations for the image pixels, a global texture model is defined for the image. Textures are built from repeating patterns, therefore the calculated texture representations are merged from pixel-level to region- 
level to formalize unique patterns, called as texture atoms, representing the image. The number of these atoms can be set beforehand, and is usually chosen quite low, resulting in a sparse texture model of the image (the original method used 20 texture atoms, and we also use the same throughout the experiments), using the atoms to classify image regions.

From the different atoms, the salient ones are searched to find those areas of the image that draw visual attention by defining a statistical texture distinctiveness value for each atom. Passive ISAR images are different from general imagery, however the main rules still hold: more distinct regions have higher statistical texture distinctiveness. Also, in such images the target is usually close to image center, which also attracts higher visual attention, therefore these image areas require higher distinctiveness. The calculated $T$ texture map is shown in Fig. 4(b), where the higher distinctiveness is represented by lighter color. The texture map is binarized with the adaptive Otsu thresholding [19] to define the initial salient blob (Fig. $4(\mathrm{c})$ ).

To extract features representing the salient object, the first step is a robust object outline detection, which is a great challenge in case of passive ISAR images, as edges can be quite blurry. To compensate for this challenge, the keypoints of the detected salient area are extracted and salient directions are calculated based on the main orientations of the gradient in the small surroundings around the keypoints. This orientation feature is then used for an improved edge enhancement by building a structural feature map. To represent the salient object as the fusion of structural and textural features, the textural distinctiveness map is also incorporated in the proposed boundary detection model.

A modification of the Harris characteristic function [20] was introduced for noisy and high curvature boundaries [18] for keypoint extraction. Keypoints are calculated as the local maxima of the Modified Harris for Edges and Corners (MHEC) function, which is based on the eigenvalues $\left(\lambda_{1}\right.$ and $\left.\lambda_{2}\right)$ of the Harris matrix, the function looks as the following:

$$
R_{\text {mod }}=\max \left(\lambda_{1}, \lambda_{2}\right)
$$

The calculated MHEC keypoint set is shown in white in Fig. 4(d), the points are selected in the $P$ keypoint set if they have $R_{\text {mod }}$ value over an adaptive Otsu threshold. Based on the $P$ point set, features are searched for object contour enhancement. Local direction as a feature [21], [22] may facilitate contour detection by defining the main orientations where relevant edges should be searched for. To handle multiple orientation cases (such as corners) and to calculate proper direction information (not only histogram binning) on a contour level (not only pixel level), the direction feature extraction algorithm introduced in [23] was applied and then the Morphological Feature Contrast (MFC) operator [24] was used for edge detection.

The local gradient orientation density (LGOD) [25] function is calculated by analyzing the small $W_{n}(i)$ neighborhood ( $n \times n$, in our case $n=3$ ) around the keypoints in the $P$ point set, and the location of its maxima is assigned to the point as the main orientation. For the $i^{t h}$ point $\left(P_{i}\right)$ its form is:

$$
\varphi_{i}=\underset{\varphi \in[-90,+90]}{\operatorname{argmax}}\left\{\frac{1}{N_{i}} \sum_{r \in W_{n}(i)} \frac{1}{h} \cdot\left\|\nabla g_{r}\right\| \cdot \kappa\left(\frac{\varphi-\varphi_{r}^{\nabla}}{h}\right)\right\},
$$

where $\nabla g_{i}$ is the gradient vector for $P_{i}$ with $\left\|\nabla g_{i}\right\|$ magnitude and $\varphi_{i}^{\nabla}$ orientation, $N_{i}=\sum_{r \in W_{n}(i)}\left\|\nabla g_{r}\right\|$ and $\kappa$ is a Gaussian smoothing kernel with $h=0.7$ bandwidth parameter.

After $\varphi_{i}$ is calculated for all points in $P$, a $\vartheta(\varphi)$ orientation histogram is defined. Orientations belonging to the maxima of this $\vartheta(\varphi)$ histogram are assumed to be the main orientations of the salient area. To calculate these orientations, Gaussians are correlated to $\vartheta(\varphi)$ iteratively. By measuring the correlation rate between $\vartheta(\varphi)$ and the Gaussian, the iterative process stops when this rate is starting to decrease. At this point, the main orientations of the salient area are extracted.

The Morphological Feature Contrast operator [24] first distinguishes background texture and isolated salient features, and it has an extension to extract linear features in defined directions. By applying this extension in the previously extracted main orientations, the relevant features can be emphasized. By fusing this Salient Direction feature map $\left(M_{S D}\right)$ with the MHEC function $\left(R_{\text {mod }}\right)$, the structural information of the salient area is enhanced in an $S$ structural feature map, which is shown in Fig. 4(e) and is calculated as follows:

$$
S=\max \left(\max \left(0, \log \left(M_{S D}\right)\right), \max \left(0, \log \left(R_{\bmod }\right)\right)\right) .
$$

By this point, only structural information is applied for object contour detection. To also incorporate textural information, the $T$ texture map (Fig. 4(b)) is fused with the $S$ structural feature map (with weight $\gamma=0.3$ ) resulting in an improved object contour representation (Fig. 4 (f)):

$$
C=\gamma|\nabla(S(x, y))|+(1-\gamma)|\nabla(T(x, y))| .
$$

By applying adaptive Otsu thresholding on the $C$ object feature map, the binary contours are defined (Fig. $4(\mathrm{~g})$ ) for further processing steps. The 5 biggest blobs are selected, followed by the extraction of contiguous blobs and extracting their contours (Fig. 4(h)). After ordering the blobs by size, the largest one is selected as the target candidate and its main length is measured (Fig. 4 (i)).

Fig. 5 shows some examples for input images and the final results of the above described target detection and extraction steps.

\section{B. Classification}

The goal of the classification step is using extracted features to recognize targets from the same class later. Since we do not have, and it would be extremely hard to obtain a large enough dataset to train deep convolutional networks for classification, our approach was to find a method that can work with a small dataset but is able to scale to larger datasets as well. The proposed solution does not need periodic re-training, is easy to extend with new target classes, and is part of a classification process that is invariant to target rotation.

First, we take the targets extracted from the previous step, and extract their contours. For classification, the contour of 

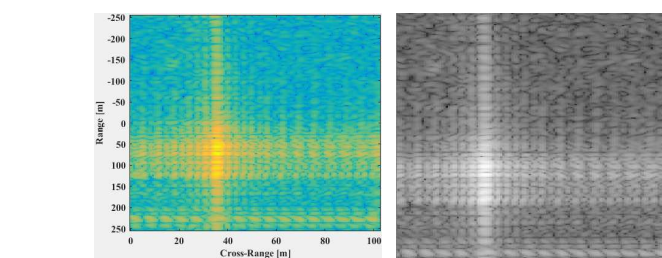

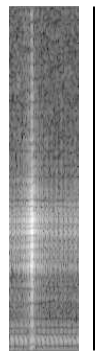

(a)

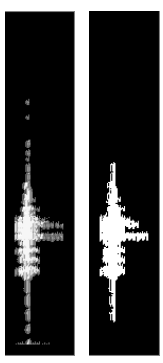

(b)

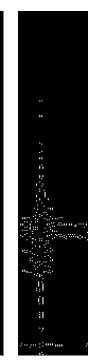

(c)

(d)

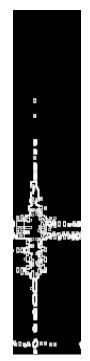

(e)

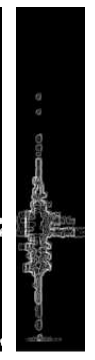

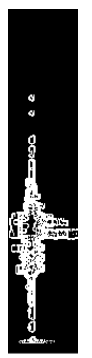

(f)

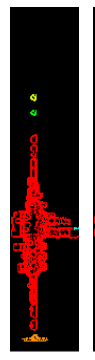

(h)

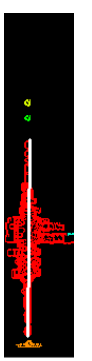

(i)
Fig. 4. The process of the segmentation and extraction, visualized.

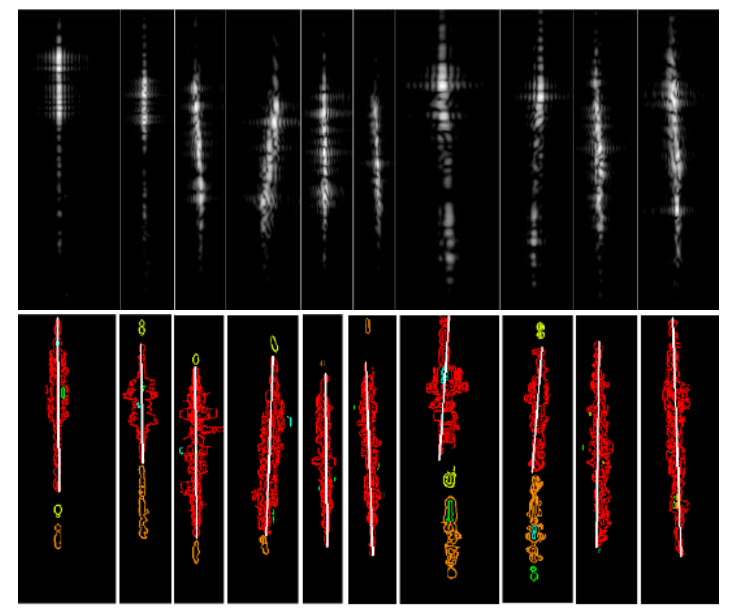

Fig. 5. Examples of input images (top) and final extracted regions and main lengths (bottom).

the candidate is transformed into a rotation invariant tangent function representation [26]. To obtain a target class estimate, we propose a method based on [16], with a point of view of content based retrieval. Using the available labeled dataset, we construct an index structure [27] which indexes the dataset based on the comparison of the extracted shape descriptors (i.e., turning function representations). The index structure is using $\mathrm{BK}^{*}$-trees described in detail in [27], in which a node can have multiple children, each child falling into a specific difference interval from its parent.

In this solution, the classification of a target becomes a content based retrieval step: an input target is a query, and we find the most similar nodes in the index structure, assigning the class of the most similar results as the class of the queried target.

Because of the index tree's structure, when looking for similar nodes and traversing the tree, large parts of the index can be disregarded at every node. This structure has multiple benefits: it is easy to extend with new elements, which only need to be added to the tree, thus no full reconstruction is necessary; we can use it to not only get a single class estimate

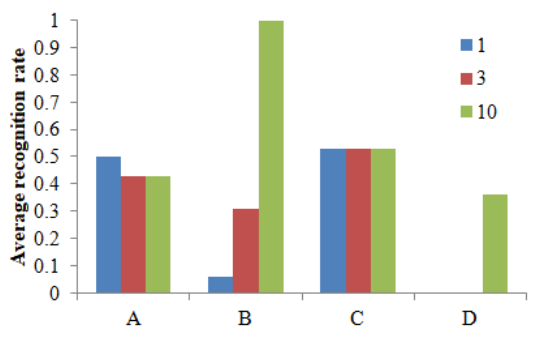

Fig. 6. Comparison of the first (1), majority of first three (3) and majority of first ten (10) approaches (for classes A-D).

TABLE II. NORMALIZED CONFUSION MATRIX.

\begin{tabular}{c|cccccccc} 
& $\mathrm{A}$ & $\mathrm{B}$ & $\mathrm{C}$ & $\mathrm{D}$ & $\mathrm{E}$ & $\mathrm{F}$ & $\mathrm{G}$ & $\mathrm{H}$ \\
\hline $\mathrm{A}$ & 0.64 & 0.14 & 0.21 & 0.00 & 0.00 & 0.00 & 0.00 & 0.00 \\
$\mathrm{~B}$ & 0.00 & 0.38 & 0.06 & 0.13 & 0.00 & 0.19 & 0.00 & 0.25 \\
$\mathrm{C}$ & 0.13 & 0.00 & 0.67 & 0.07 & 0.07 & 0.00 & 0.00 & 0.07 \\
$\mathrm{D}$ & 0.00 & 0.27 & 0.18 & 0.55 & 0.00 & 0.00 & 0.00 & 0.00 \\
$\mathrm{E}$ & 0.00 & 0.00 & 0.00 & 0.00 & 0.56 & 0.00 & 0.33 & 0.11 \\
$\mathrm{~F}$ & 0.00 & 0.17 & 0.06 & 0.06 & 0.06 & 0.28 & 0.33 & 0.06 \\
$\mathrm{G}$ & 0.00 & 0.00 & 0.00 & 0.00 & 0.07 & 0.00 & 0.93 & 0.00 \\
$\mathrm{H}$ & 0.00 & 0.06 & 0.00 & 0.00 & 0.00 & 0.06 & 0.06 & 0.83
\end{tabular}

for a query target, but to obtain the first $\mathrm{N}$ most similar candidates as well, being able to keep a constant statistics of the class estimates and propose the most frequent estimate as the target class; it is also a very lightweight solution, a class estimation step requiring less than $0.2 \mathrm{~s}(1.6 \mathrm{GHz}$ Core i5); it is also easy to parallelize, since we can run multiple retrieval steps on the index in parallel, thus multiple targets can be classified simultaneously.

Using the shape representation of an extracted target, we classify the target by performing a retrieval step on the available index and taking the best results as an indicator of the class.

\section{Evaluation}

As mentioned above, we used a dataset of 128 real passive ISAR range-crossrange images of 8 targets, 2 aerial (planes), 6 nautical (ships). We label these classes with letters A to $\mathrm{H}$.

Using the above described index structure, we performed retrievals on the indexed dataset to find target classes. For evaluation, we indexed the dataset and performed retrievals for each dataset element, discarding the first result (which was always the input/query image).

First, we used a smaller subset (classes A-D, containing 56 samples), to perform retrievals where we evaluated the first best match, the majority of the first 3 matches, and the majority of the first 10 matches to obtain a class estimate. Fig. 6 shows the average recognition rates for these retrievals: the class estimate of a target gets better if we take into consideration not only the closest match, but a statistics of matches. In practice this means, that when we have more samples of the same target class incorporated in the index structure, the recognition of a target class will improve (i.e., the more we see the same class, the better we will be able to recognize it).

For our evaluations, we used the third approach: for each queried target image, we retrieve the 10 closes matches and take the majority of the results as the class estimate. Table (II) shows the normalized confusion values of the classification 


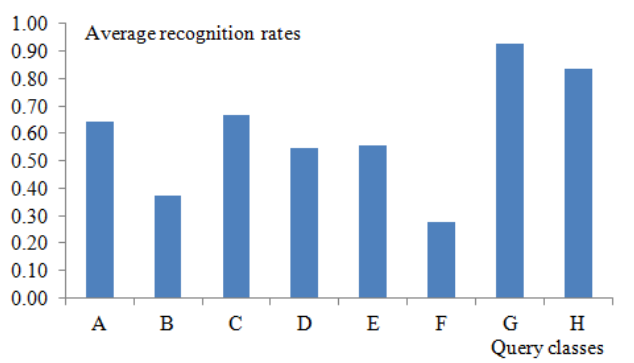

Fig. 7. Average recognition rates for the proposed method.

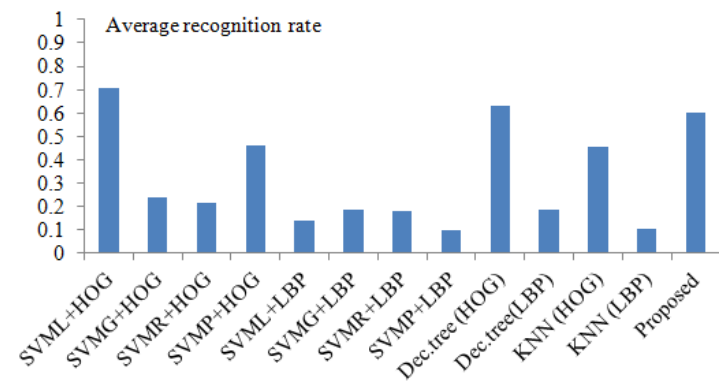

Fig. 8. Average recognition rates for the SVM approaches, the decision tree and $\mathrm{kNN}$ methods and the proposed method.

using the full dataset, and Fig. 7 shows the average recognition rates for the used classes. From these results we can see that some classes were well recognized (94\%), others had a lower recognition rate $(28 \%)$, the average recognition rate being $61 \%$.

To put these results into perspective, we also evaluated other classification methods on the same dataset. First, we ran SVM (support vector machine) classifications, using histogram of oriented gradients and local binary pattern features, and we show the classification results in Fig. 8 We used the Matlab SVM implementation and tried linear (SVML), Gaussian (SVMG), RBF (SVMR) and polynomial (SVMP) kernels. We also tried decision tree (Dec.tree) and $\mathrm{k}$ nearest neighbor $(\mathrm{kNN})$ learner templates. All versions were run 10 times, with random $75 \%$ of the dataset used for training and $25 \%$ for testing, and averaging the results. The results show that the best SVM average (SVML+HOG: $70 \%$ ) is similar to our proposed results.

However, we also measured training/indexing and prediction/retrieval times for all methods, and we show in Table III the results of those methods that are close to the proposed in average recognition rates, namely HOG-based SVM, decision trees and kNN. Compared to methods with similar recognition rate, the proposed method is more lightweight and faster both in indexing and in retrieval. We also need to keep in mind here, that the proposed approach only needs to build the index once (with later elements added to the index tree), while for the others training needs to be performed repeatedly (with increased time) when new target classes/elements need to be added to the model.

To showcase another benefit and strength of the proposed approach, we performed another evaluation step. We created 2 rotated (with 45 and 135 degrees) versions of 1 raw input image from each class (16 images in total), and tried to classify these rotated versions using the proposed approach and the
TABLE III. INDEXING/TRAINING AND CLASSIFICATION/PREDICTION TIMES (S) FOR THE PROPOSED METHOD, SVM, DECISION TREES AND KNN.

\begin{tabular}{|c|c|c|}
\hline Methods & Indexing/training (s) & Classification/prediction (s) \\
\hline \hline Proposed & 2.87 & 0.20 \\
\hline SVML+HOG & 17.39 & 1.79 \\
\hline SVMP+HOG & 58.38 & 8.63 \\
\hline Dec.tree(HOG) & 107.12 & 0.11 \\
\hline KNN(HOG) & 23.02 & 16.30 \\
\hline
\end{tabular}

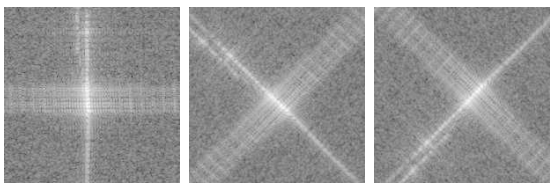

(a)
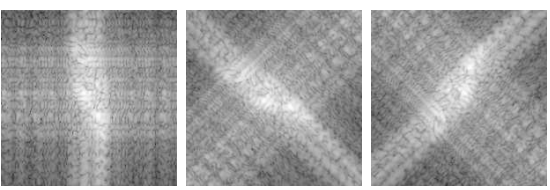

(b)

Fig. 9. Samples of artificially rotated raw inputs.

closest methods from Fig. 8. The rotated images were not included in the indexing and in the model training steps, only used as unknown input targets. Fig. 9 shows some examples of such rotated images. The goal of this evaluation step is to show that the proposed method is strong in recognizing the class of targets which are rotated versions of targets seen before (i.e., have samples of the target in the index, but from different angles). Fig. 10 shows average recognition rates for the rotated inputs ( 2 images for each class, averaged). The results show that the proposed method could correctly classify the rotated targets, while the other approaches mostly failed.

As final examples, Fig. 11 shows two examples of input passive ISAR range-crossrange images (with target regions zoomed in) and the first 3 matches from the index.

\section{CONCLUSION}

In this paper we presented an automatic target extraction and classification method for passive multistatic ISAR rangecrossrange images, to show the possibility and capability of image feature based approaches for such tasks. The presented approach handles the classification from a content based retrieval point of view, providing several benefits: can work with a small number of samples, moreover, it is easy to extend with more data; it is lightweight and can handle multi-target classification as well; does not need re-training as traditional machine learning approaches; it can handle the classification of rotated targets; its robustness can be increased by incorporating more variations of class samples. The proposed approach is lightweight enough to be embeddable to existing ATR systems that incorporate passive multistatic ISAR imaging. In the future we hope to further increase the robustness and speed of the approach.

\section{ACKNOWLEDGMENT}

This work was partially funded by the European Defence Agency (EDA) in the framework of the Multichannel passive 


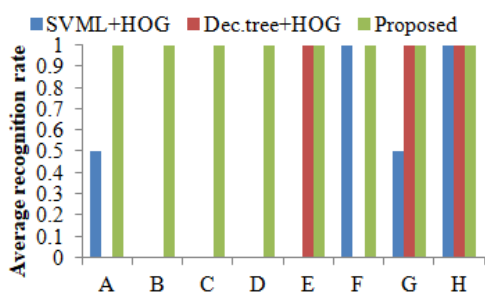

Fig. 10. Average recognition rates for the rotated inputs for the 8 classes $(\mathrm{A}-\mathrm{H})$.

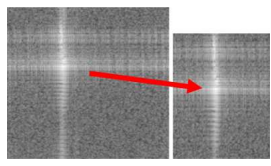

(a)
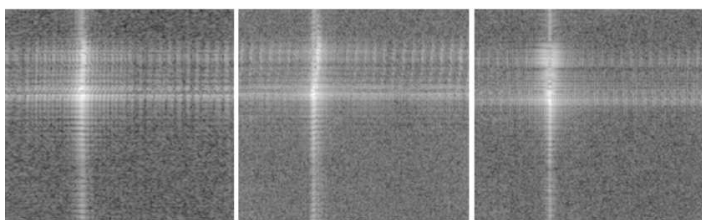

(b)

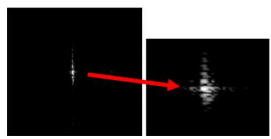

(c)
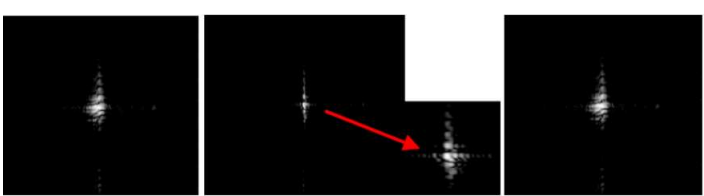

(d)

Fig. 11. Examples for queries (a, c) and first 3 responses (b, d).

ISAR imaging for military applications (MAPIS) R\&T project, and by the Hungarian National Research, Development and Innovation Fund (NKFIA) grant nr. KH-125681

\section{REFERENCES}

[1] D. Olivadese, E. Giusti, D. Petri, M. Martorella, A. Capria, and F. Berizzi, "Passive ISAR with DVB-T signals," IEEE Tr. on Geoscience and Remote Sensing, vol. 51, no. 8, pp. 4508-4517, 2013.

[2] P. Krysik, K. Kulpa, P. Samczynski, K. Szumski, and J. Misiurewicz, "Moving target detection and imaging using GSM-based passive radar," in Proc. of IET Intl. Conf. on Radar Systems, 2012.

[3] C. Coleman and H. Yardley, "Passive bistatic radar based on target illuminations by digital audio broadcasting," IET Radar, Sonar \& Navigation, vol. 2, no. 5, pp. 366-375, 2008.

[4] A. Capria, E. Giusti, C. Moscardini, M. Conti, D. Petri, M. Martorella, and F. Berizzi, "Multifunction imaging passive radar for harbour protection and navigation safety," IEEE Aerospace and Electronic Systems Magazine, vol. 32, no. 2, pp. 30-38, 2017.

[5] J. L. Garry, C. J. Baker, G. E. Smith, and R. L. Ewing, "Investigations toward multistatic passive radar imaging," in Proc. of IEEE Radar Conference, 2014.

[6] P. Samczynski, K. Kulpa, M. Baczyk, and D. Gromek, "SAR/ISAR imaging in passive radars," in Proc. of IEEE Radar Conference, 2016, pp. $1859-865$.
[7] D. Gromek, M. Baczyk, P. Samczynski, K. Kulpa, and P. Baranowski, "A concept of the parametric autofocus method for passive ISAR imaging," in Proc. of Intl. Radar Symposium, 2017.

[8] W. Qiu, E. Giusti, A. Bacci, M. Martorella, F. Berizzi, H. Zhao, and $\mathrm{Q}$. Fu, "Compressive sensing-based algorithm for passive bistatic ISAR with DVB-T signals," IEEE Tr. on Aerospace and Electronic Systems, vol. 51, no. 3, pp. 2166-2180, 2015.

[9] M.Martorella and E. Giusti, "Theoretical foundation of passive bistatic ISAR imaging," IEEE Tr. on Aerospace and Electronic Systems, vol. 50, no. 3, pp. 1647-1659, 2014.

[10] J. Pisane, S. Azarian, M. Lesturgie, and J. Verly, "Automatic target recognition for passive radar," IEEE Tr. on Aerospace and Electronic Systems, vol. 50, no. 1, pp. 371-392, 2014.

[11] L. M. Ehrman and A. D. Lanterman, "Automated target recognition using passive radar and coordinated flight models," in Proc. of Automatic Target Recognition XIII (SPIE 5094), 2003.

[12] C. Benedek and M. Martorella, "Moving target analysis in ISAR image sequences with a multiframe Marked Point Process Model," IEEE Tr. on Geoscience and Remote Sensing, vol. 52, no. 4, pp. 2234-2246, 2014.

[13] _ "Ship structure extraction in isar image sequences by a markovian approach," in Proc. of IET Intl. Conf. on Radar Systems, 2012, pp. 6266.

[14] A. Manno-Kovacs, "Direction selective vector field convolution for contour detection," in Proc. of IEEE Intl. Conf. on Image Processing (ICIP), 2014, pp. 4722-4726.

[15] A. Kovacs and T. Szirányi, "Harris function based active contour external force for image segmentation," Pattern Recognition Letters, vol. 33, no. 9, pp. 1180-1187, 2012.

[16] L. Kovács, A. Kovacs, A. Utasi, and T. Szirányi, "Flying target detection and recognition by feature fusion," Opical Engineering, vol. 51, no. 11, p. 117002,2012

[17] M. Martorella, "Novel approach for ISAR image cross-range scaling," IEEE Tr. on Aerospace and Electronic Systems, vol. 41, no. 1, pp. 281294, 2008.

[18] C. Scharfenberger, A. Wong, K. Fergani, J. S. Zelek, and D. Clausi, "Statistical textural distinctiveness for salient region detection in natural images," in Proc. of IEEE Intl. Conf. on Computer Vision and Pattern Recognition (CVPR), 2013, pp. 979-986.

[19] N. Otsu, "A threshold selection method from gray-level histograms," IEEE Tr. on Systems, Man and Cybernetics, vol. 9, no. 1, pp. 62-66, 1979.

[20] C. Harris and M. Stephens, "A combined corner and edge detector," in Proc. of the 4th Alvey Vision Conf., 1988, pp. 147-151.

[21] P. Perona, "Orientation diffusions," IEEE Tr. on Image Processing, vol. 7, no. 3, pp. 457-467, 1998.

[22] S. Yi, D. Labate, G. R. Easley, and H. Krim, "A shearlet approach to edge analysis and detection," IEEE Tr. on Image Processing, vol. 18, no. 5, pp. 929-941, 2009.

[23] A. Manno-Kovacs and T. Szirányi, "Orientation-selective building detection in aerial images," ISPRS J. of Photogrammetry and Remote Sensing, vol. 108, pp. 94-112, 2015.

[24] I. Zingman, D. Saupe, and K. Lambers, "A morphological approach for distinguishing texture and individual features in images," Pattern Recognition Letters, vol. 47, pp. 129-138, 2014.

[25] C. Benedek, X. Descombes, and J. Zerubia, "Building development monitoring in multitemporal remotely sensed image pairs with stochastic birth-death dynamics," IEEE Tr. on Pattern Analysis and Machine Intelligence, vol. 34, no. 1, pp. 33-50, 2012.

[26] L. J. Latecki and R. Lakamper, "Application of planar shape comparison to object retrieval in image databases," Pattern Recognition, vol. 35, no. 1, pp. 15-29, 2002.

[27] L. Kovács, "Parallel multi-tree indexing for evaluating large descriptor sets," in Proc. of IEEE Intl. Workshop on Content-Based Multimedia Indexing (CBMI), 2013, pp. 173-178. 